\title{
Practical exponential stability of impulsive stochastic functional differential equations
}

\author{
Tomás Caraballo $^{a}$, Mohamed Ali Hammami ${ }^{b}$, Lassaad Mchiri ${ }^{b}$ \\ ${ }^{a}$ Universidad de Sevilla, Dpto. Ecuaciones Diferenciales y Análisis Numérico, Facultad de Matemáticas, C/ Tarfia s/n, \\ 41012-Sevilla (Spain). E-mail: caraball@us.es \\ ${ }^{b}$ University of Sfax, Faculty of Sciences of Sfax, Department of Mathematics, Tunisia. E-mail: MohamedAli.Hammami@fss.rnu.tn
}

\begin{abstract}
This paper is devoted to the investigation of the practical exponential stability of impulsive stochastic functional differential equations. The main tool used to prove the results is the Lyapunov-Razumikhin method which has proven very useful in dealing with stability problems for differential systems when the delays involved in the equations are not differentiable but only continuous. An illustrative example is also analyzed to show the applicability and interest of the main results.
\end{abstract}

Key words: Impulsive stochastic functional differential equations, almost sure practical exponential stability, Lyapunov-Razumikhin method.

2000 MSC: 93E03, 60H10.

\section{Introduction}

Impulsive differential equations are suitable for the mathematical simulation of evolutionary processes in which some parameters may undergo relatively long periods of smooth variation followed by a short-term rapid change (that is, jumps) in their values. Processes of this type are often investigated in various fields of science and technology (see [11]).

Needless to say that, in general, the evolution of a dynamical system may not only be determined by its current state. It is sensible to admit that its past or history may also have some influence in its future evolution. This fact justifies the appearance of several types of delay or memory in the models. Stochastic functional differential equations are often used as appropriate tools for the description and analysis of such systems, see [16]-[17].

Nowadays, impulsive deterministic and stochastic functional differential equations represent an emerging field drawing attention from both theoretical and applied disciplines, and which has been successfully applied to problems in mechanics, electricity, economics, physics and several fields in engineering (see, e.g. [5][12] for more details).

Over the last decades, many interesting results concerning stability of stochastic functional (and evoluPreprint submitted to Systems and Control Letters tion) differential equations have been obtained (see [7][8], [15]-[17]). In [14], Mao developed the Razumikhin method on this aspect, and established some Razumikhin-type theorems on exponential stability of stochastic functional differential equations for finitedimensional stochastic ordinary differential systems with delays. The advantage of this method, in contrast to the classical Lyapunov one, is that it is not necessary to use functionals to analyze the stability. Instead, one can consider Lyapunov functions and, additionally, the type of delays that can be handled is more general. For instance, if the problem contains variable delays, these only need to be continuous while to apply the classical Lyapunov theory (see [4]), the variable delay function is required to be continuously differentiable. Some results concerning stability of impulsive stochastic functional differential equations (using the Razumikhin method) have been proved in [5], [9]-[10], [18]-[19] and [12].

However, when the origin is not necessarily an equilibrium point, it is still possible to analyze the asymptotic stability of solutions with respect to a small neighborhood of the origin, which yields the concept of practical stability (see [6] and the references therein). It is worth mentioning some previous works on practical stability in the deterministic and stochastic frameworks (see $[1,2,3,6])$. In particular, we initiated very recently the study of this practical stability for non-delay

August 29, 2017 
stochastic partial differential equations in [1], [2]. Notice that inequality (2.2) (see Section 2 below) implies that $x(t)$ is ultimately bounded by a small bound $r>0$, that is, $|x(t)|$ is small for sufficiently large $t$. This can be viewed as a robustness property of almost sure convergence to the origin provided that $f$ and $g$ satisfy $f(0, t)=0$ and $g(0, t)=0$, for all $t \geq 0$. In this case the origin becomes an equilibrium point.

The content of the paper is as follows. In Section 2 we introduce the basic notations and assumptions. Section 3 is devoted to prove some sufficient conditions ensuring practical $p$-th moment exponential and almost sure exponential stability of solutions to impulsive stochastic functional differential equations. We prove a sufficient condition ensuring the convergence of solutions to a ball with radius $\eta>0$, even in the case that zero is not an equilibrium point. Finally, two examples to illustrate our abstract theory are included in Section 4.

\section{Preliminaries}

For $x \in \mathbb{R}^{n},|x|$ denotes the Euclidian norm of $x$ and $[x]$ the biggest integer less than or equal to $x$. For $-\infty<a<b<\infty$, we say that a function from $[a, b]$ to $\mathbb{R}^{n}$ is piecewise continuous, if the function has at most a finite number of jump discontinuities on $(a, b]$ and is continuous from the right for all points in $[a, b)$. Given $\tau>0, \mathcal{P C}\left([-\tau, 0] ; \mathbb{R}^{n}\right)$ denotes the family of piecewise continuous functions from $[-\tau, 0]$ to $\mathbb{R}^{n}$. A norm on $\mathcal{P C}\left([-\tau, 0] ; \mathbb{R}^{n}\right)$ is defined as $\|\varphi\|=\sup _{-\tau \leq \theta \leq 0}|\varphi(\theta)|$ for $\varphi \in \mathcal{P C}\left([-\tau, 0] ; \mathbb{R}^{n}\right)$.

Let $(\Omega, \mathcal{F}, \mathbb{P})$ be a complete probability space with a filtration $\left\{\mathcal{F}_{t}\right\}_{t \geq 0}$ satisfying the usual conditions and $W(t)=$ $\left(W_{1}(t), W_{2}(t), \ldots, W_{m}(t)\right)$ be an $m$-dimensional standard Wiener process defined on $(\Omega, \mathcal{F}, \mathbb{P})$ and adapted to $\left\{\mathcal{F}_{t}\right\}_{t \geq 0}$. For $p>0$ and $t \geq 0$, let $L_{\mathcal{F}}^{p}\left([-\tau, 0], \mathbb{R}^{n}\right)$ denote the family of all $\mathcal{F}_{t}$-measurable $\mathcal{P C}\left([-\tau, 0] ; \mathbb{R}^{n}\right)$-valued random processes $\varphi=\{\varphi(-\theta):-\tau \leq \theta \leq 0\}$ such that $\sup _{-\tau \leq \theta \leq 0} \mathbb{E}|\varphi(\theta)|^{p}<\infty$. And let $L_{\mathcal{F}_{t}}^{p}\left(\Omega, \mathbb{R}^{n}\right)$ denote the family of all $\mathcal{F}_{t}$-measurable $\mathbb{R}^{n}$-valued random variables $X$, such that $\mathbb{E}|X|^{p}<\infty$. Finally, let $\mathcal{P} C^{b}\left([-\tau, 0] ; \mathbb{R}^{n}\right)$ denote the family of all bounded $\mathcal{P C}\left([-\tau, 0] ; \mathbb{R}^{n}\right)$-valued functions.

In this paper, we consider the following impulsive stochastic functional differential equation:

$$
\left\{\begin{aligned}
d x(t) & =f\left(x_{t}, t\right) d t+g\left(x_{t}, t\right) d W(t), \quad t \neq t_{k}, t \geq t_{0}, \\
\Delta x\left(t_{k}\right) & =I_{k}\left(x\left(t_{k}^{-}\right), t_{k}\right), \quad k \in \mathbb{N}, \\
x_{t_{0}} & =\xi, \text { i.e. } \quad x\left(t_{0}+\theta\right)=\xi(\theta), \theta \in[-\tau, 0]
\end{aligned}\right.
$$

where the initial value $\xi \in \mathcal{P} C^{b}\left([-\tau, 0] ; \mathbb{R}^{n}\right)$, $x(t)=\left(x_{1}(t), \ldots, x_{n}(t)\right)^{T}, x_{t} \in L_{\mathcal{F}_{t}}^{p}\left([-\tau, 0], \mathbb{R}^{n}\right), f:$
$L_{\mathcal{F}_{t}}^{p}\left([-\tau, 0], \mathbb{R}^{n}\right) \times \mathbb{R}_{+} \longrightarrow \mathbb{R}^{n}, g: L_{\mathcal{F}_{t}}^{p}\left([-\tau, 0], \mathbb{R}^{n}\right) \times$ $\mathbb{R}_{+} \longrightarrow \mathbb{R}^{n \times m}, I_{k}(\cdot, \cdot): \mathbb{R}^{n} \times \mathbb{R}_{+} \stackrel{\mathcal{F}_{t}}{\longrightarrow} \mathbb{R}^{n}$, where $I_{k}\left(x\left(t_{k}^{-}\right), t_{k}\right)$ represents the impulsive perturbation of $x$ at time $t_{k}$, and we suppose that there exists $t \geq t_{0}$ such that $f(0, t) \neq 0, g(0, t) \neq 0$ and $I_{k}(0, t) \neq 0, k \in \mathbb{N}$.

The fixed moments of impulse times $t_{k}$ satisfy $0 \leq$ $t_{0}<t_{1}<\ldots<t_{k}<\ldots, t_{k} \rightarrow \infty($ as $k \rightarrow \infty), \Delta x\left(t_{k}\right)=$ $x\left(t_{k}\right)-x\left(t_{k}^{-}\right)$.

As standing hypotheses, we assume that $f(\varphi, t)$ and $g(\varphi, t)$ are continuous for almost all $t \in\left[t_{0}, \infty\right)$ and are Lipschitz in $\varphi$ in each compact set in $L_{\mathcal{F}_{t}}^{p}\left([-\tau, 0], \mathbb{R}^{n}\right)$; $I_{k}(x, t)$ is Lipschitz in $x$ for all $x \in \mathbb{R}^{n}, k \in \mathbb{N}$.

As we are interested in the asymptotic behavior of the solutions, we assume that for any $\xi \in \mathcal{P} C^{b}\left([-\tau, 0] ; \mathbb{R}^{n}\right)$, there exists a unique stochastic process, denoted by $x\left(t, t_{0}, \xi\right)$, satisfying system (2.1), which is continuous on the right-hand side and possessing limit on the lefthand side. For some results on the existence and uniqueness of solutions of (2.1) the reader is referred to [13].

Now we will establish the definition of $p$-moment and almost sure practical stability and recall the definition of generating operator for our equation.

Definition 2.1. For $p>0$, system (2.1) is said to be $p$ th moment practically exponentially stable if there exist positive constants $\lambda, C$ and $\eta$ such that

$$
\mathbb{E}\left|x\left(t, t_{0}, \xi\right)\right|^{p} \leq C\|\xi\|^{p} e^{-\lambda\left(t-t_{0}\right)}+\eta, \quad t \geq t_{0},
$$

for all $\xi \in \mathcal{P} C^{b}\left([-\tau, 0] ; \mathbb{R}^{n}\right)$

Definition 2.2. The ball $B_{\eta}:=\left\{x \in \mathbb{R}^{n}:|x| \leq \eta\right\}, \eta>$ 0 , is said to be almost surely practically exponentially stable if, for all $\xi \in \mathcal{P} C^{b}\left([-\tau, 0] ; \mathbb{R}^{n}\right)$ and every $t \geq t_{0}$,

$$
\left|x\left(t, t_{0}, \xi\right)\right| \leq C\|\xi\|^{p} e^{-\lambda\left(t-t_{0}\right)}+\eta, \text { a.s. }
$$

System (2.1) is said to be almost surely practically exponentially stable if there exists $\eta>0$ such that $B_{\eta}$ is almost surely practically exponentially stable.

Definition 2.3. Let $C^{2,1}\left(\mathbb{R}^{n} \times\left[t_{0}-\tau, \infty\right) ; \mathbb{R}_{+}\right)$denote the family of all nonnegative functions $V(x, t)$ on $\mathbb{R}^{n} \times\left[t_{0}-\tau, \infty\right)$ that are continuously twice differentiable in $x$ and once in $t$. For a function $V \in$ $C^{2,1}\left(\mathbb{R}^{n} \times\left[t_{0}-\tau, \infty\right) ; \mathbb{R}_{+}\right)$we define the operator $\mathcal{L} V: L_{\mathcal{F}_{t}}^{p}\left([-\tau, 0], \mathbb{R}^{n}\right) \times\left[t_{0}, \infty\right) \longrightarrow \mathbb{R}$ for system $(2.1)$ by

$$
\begin{aligned}
\mathcal{L} V(\varphi, t)= & V_{t}(\varphi(0), t)+V_{x}(\varphi(0), t) f(\varphi, t) \\
& +\frac{1}{2} \operatorname{trace}\left[g^{T}(\varphi, t) V_{x x}(\varphi(0), t) g(\varphi, t)\right],
\end{aligned}
$$

where $\varphi \in L_{\mathcal{F}_{t}}^{p}\left([-\tau, 0], \mathbb{R}^{n}\right)$ and 


$$
\begin{aligned}
V_{t}(x, t) & =\frac{\partial V}{\partial t}(x, t) ; V_{x}(x, t)=\left(\frac{\partial V}{\partial x_{1}}(x, t), \ldots, \frac{\partial V}{\partial x_{n}}(x, t)\right) ; \\
V_{x x}(x, t) & =\left(\frac{\partial^{2} V}{\partial x_{i} \partial x_{j}}(x, t)\right)_{n \times n} .
\end{aligned}
$$

If we replace $\varphi$ in the expression (2.3) by a segment solution $x_{t}$ of system (2.1), then it becomes

$$
\begin{aligned}
\mathcal{L} V\left(x_{t}, t\right)= & V_{t}(x(t), t)+V_{x}(x(t), t) f\left(x_{t}, t\right) \quad(2.4) \\
& +\frac{1}{2} \operatorname{trace}\left[g^{T}\left(x_{t}, t\right) V_{x x}(x(t), t) g\left(x_{t}, t\right)\right] .
\end{aligned}
$$

\section{Main results}

In this section, we first investigate the $p$-th moment practical exponential stability and the almost sure practical exponential stability of system (2.1) by using the Razumikhin method. The results show that the impulsive effects can produce some kind of stabilization on the system and achieve $p$-th moment practical exponential stability.

Theorem 3.1. Assume that there exist a function $V \in$ $C^{2,1}\left(\mathbb{R}^{n} \times\left[t_{0}-\tau, \infty\right) ; \mathbb{R}_{+}\right)$and positive constants $p, c, c_{1}$, $c_{2}, c_{3}, \lambda, h, \psi, \rho_{k}, k \in \mathbb{N}, \delta \geq 0$ and $\gamma \geq 1$ such that

(i) $c_{1}|x|^{p} \leq V(x, t) \leq c_{2}|x|^{p}+c_{3}, \quad \forall(x, t) \in \mathbb{R}^{n} \times\left[t_{0}-\right.$ $\tau, \infty)$

(ii) $\quad \mathbb{E} V\left(x+I_{k}\left(x, t_{k}\right), t_{k}\right) \leq d_{k} \mathbb{E} V\left(x, t_{k}^{-}\right)+\rho_{k}, \quad \forall k \in$ $\mathbb{N}, x \in L_{\mathcal{F}_{t}}^{p}\left(\Omega, \mathbb{R}^{n}\right)$, where $0<d_{k} \leq 1,0<\rho_{k} \leq d$ with $d=\sup _{k \in \mathbb{N}} \rho_{k}<\infty$.

(iii) $\mathbb{E} \mathcal{L} V(\varphi, t) \leq c \mathbb{E} V(\varphi(0), t)+\psi, \forall t \geq t_{0}, t \neq t_{k}, k \in$ $\mathbb{N}$ and $\varphi \in L_{\mathcal{F}_{t}}^{p}\left([-\tau, 0], \mathbb{R}^{n}\right)$, whenever

$$
\mathbb{E} V(\varphi, t+\theta)<q \mathbb{E} V(\varphi(0), t)+\delta, \quad \theta \in[-\tau, 0],
$$

where $q \geq \gamma e^{\lambda \tau}$ is a constant.

(iv) $\gamma \geq \max \left\{\frac{1}{d_{k}}, e^{(\lambda+c)\left(t_{1}-t_{0}\right)}\right\}, \quad \ln d_{k} \leq-(\lambda+c)\left(t_{k+1}-\right.$ $\left.t_{k}\right), k \in \mathbb{N}$, and

$$
\Delta_{\text {sup }}=\sup _{k \in \mathbb{N}}\left\{t_{k+1}-t_{k}\right\} \leq h<\infty, \quad e^{c h} \leq \frac{c_{3} \gamma}{\psi h+c_{3}} e^{\lambda \tau} .
$$

Then, system (2.1) is p-th moment practically exponentially stable.

Proof. Given an initial datum $\xi \in \mathcal{P} C^{b}\left([-\tau, 0] ; \mathbb{R}^{n}\right)$, we write $x\left(t, t_{0}, \xi\right)=x(t)$ for short. Choose $M>0$ such that

$$
0<c_{2} e^{(\lambda+c)\left(t_{1}-t_{0}\right)} \leq M \leq c_{2} \gamma e^{\lambda \tau} .
$$

Then, by condition ( $i$, we have for $t \in\left[t_{0}-\tau, t_{0}\right]$

$$
\mathbb{E} V(x(t), t) \leq c_{2}\|\xi\|^{p}+c_{3}<M\|\xi\|^{p} e^{-\lambda\left(t_{1}-t_{0}\right)}+r,
$$

where $\left(\psi h+c_{3}\right) e^{c h} \leq r \leq c_{3} \gamma e^{\lambda \tau}$. Next, we shall prove that, for any $k \in \mathbb{N}$ and $t \in\left[t_{k-1}, t_{k}\right.$ ),

$$
\mathbb{E} V(x(t), t) \leq M\|\xi\|^{p} e^{-\lambda\left(t_{k}-t_{0}\right)}+r .
$$

We proceed by induction and split our proof into several steps.

Step 1:

Arguing by contradiction, we first prove that

$$
\mathbb{E} V(x(t), t) \leq M\|\xi\|^{p} e^{-\lambda\left(t_{1}-t_{0}\right)}+r, \quad t \in\left[t_{0}, t_{1}\right) .
$$

Indeed, let us assume that inequality (3.4) is not true. Then, there exists some $t \in\left[t_{0}, t_{1}\right)$ such that $\mathbb{E} V(x(t), t)>M\|\xi\|^{p} e^{-\lambda\left(t_{1}-t_{0}\right)}+r$.

Define $t^{*}=\inf \left\{t \in\left[t_{0}, t_{1}\right) ; \mathbb{E} V(x(t), t)>M\|\xi\|^{p} e^{-\lambda\left(t_{1}-t_{0}\right)}+r\right\}$.

Thanks to (i) and the Lebesgue dominated convergence theorem, the mapping $t \in\left[t_{0}, t_{1}\right) \mapsto \mathbb{E} V(x(t), t)$ is continuous. Then

$$
\begin{aligned}
\lim _{t \rightarrow t_{0}^{-}} \mathbb{E} V(x(t), t) & =\lim _{t \rightarrow t_{0}^{+}} \mathbb{E} V(x(t), t) \\
& =\mathbb{E} V\left(x\left(t_{0}\right), t_{0}\right) \\
& <M\|\xi\|^{p} e^{-\lambda\left(t_{1}-t_{0}\right)}+r .
\end{aligned}
$$

Therefore, $t^{*} \in\left(t_{0}, t_{1}\right)$ and

$$
\begin{gathered}
\mathbb{E} V\left(x\left(t^{*}\right), t^{*}\right)=M\|\xi\|^{p} e^{-\lambda\left(t_{1}-t_{0}\right)}+r, \\
\mathbb{E} V(x(t), t)<M\|\xi\|^{p} e^{-\lambda\left(t_{1}-t_{0}\right)}+r, \quad t \in\left[t_{0}-\tau, t^{*}\right) .
\end{gathered}
$$

Define $t_{*}=\sup \left\{t \in\left[t_{0}, t^{*}\right] ; \mathbb{E} V(x(t), t) \leq c_{2}\|\xi\|^{p}+c_{3}\right\}$, then $t_{*} \in\left[t_{0}, t^{*}\right)$ and by the continuity of $\mathbb{E} V(x(\cdot), \cdot)$,

$$
\begin{gathered}
\mathbb{E} V\left(x\left(t_{*}\right), t_{*}\right)=c_{2}\|\xi\|^{p}+c_{3}, \\
\mathbb{E} V(x(t), t)>c_{2}\|\xi\|^{p}+c_{3}, \quad t \in\left(t_{*}, t^{*}\right] .
\end{gathered}
$$

Consequently, for all $t \in\left[t_{*}, t^{*}\right]$, we have

$$
\begin{aligned}
\mathbb{E} V(x(t+\theta), t+\theta) & \leq M\|\xi\|^{p} e^{-\lambda\left(t_{1}-t_{0}\right)}+r \\
& \leq c_{2} \gamma e^{\lambda \tau}\|\xi\|^{p} e^{-\lambda\left(t_{1}-t_{0}\right)}+r \\
& <\gamma e^{\lambda \tau}\left(c_{2}\|\xi\|^{p}+\frac{r}{\gamma} e^{-\lambda \tau}\right) \\
& \leq \gamma e^{\lambda \tau} \mathbb{E} V\left(x\left(t_{*}\right), t_{*}\right) \\
& \leq q \mathbb{E} V\left(x\left(t_{*}\right), t_{*}\right) .
\end{aligned}
$$

Then, for all $\theta \in[-\tau, 0]$,

$$
\mathbb{E} V(x(t+\theta), t+\theta)<q \mathbb{E} V(x(t), t)+\delta .
$$

By the Razumikhin-type condition (iii),

$$
\mathbb{E} \mathcal{L} V\left(x_{t}, t\right) \leq c \mathbb{E} V(x(t), t)+\psi, \quad t \in\left[t_{*}, t^{*}\right] .
$$


Applying the Itô formula on $\left[t_{*}, t^{*}\right]$

$$
\begin{aligned}
& \mathbb{E} V\left(x\left(t^{*}\right), t^{*}\right) \\
& =\mathbb{E} V\left(x\left(t_{*}\right), t_{*}\right)+\int_{t_{*}}^{t^{*}} \mathbb{E} \mathcal{L} V\left(x_{s}, s\right) d s \\
& \leq \mathbb{E} V\left(x\left(t_{*}\right), t_{*}\right)+c \int_{t_{*}}^{t^{*}} \mathbb{E} V(x(s), s) d s+\psi\left(t^{*}-t_{*}\right) \\
& \leq \mathbb{E} V\left(x\left(t_{*}\right), t_{*}\right)+c \int_{t_{*}}^{t^{*}} \mathbb{E} V(x(s), s) d s+\psi \Delta_{\text {sup }},
\end{aligned}
$$

and thus,

$$
\begin{aligned}
\mathbb{E} V\left(x\left(t^{*}\right), t^{*}\right) \leq & \mathbb{E} V\left(x\left(t_{*}\right), t_{*}\right) \\
& +c \int_{t_{*}}^{t^{*}} \mathbb{E} V(x(s), s) d s+\psi h .
\end{aligned}
$$

By (3.1), (3.5), (3.7) and the Gronwall inequality,

$$
\begin{aligned}
\mathbb{E} V\left(x\left(t^{*}\right), t^{*}\right) & \leq\left(\mathbb{E} V\left(x\left(t_{*}\right), t_{*}\right)+\psi h\right) e^{c\left(t^{*}-t_{*}\right)} \\
& <\left(c_{2}\|\xi\|^{p}+c_{3}+\psi h\right) e^{c\left(t_{1}-t_{0}\right)} \\
& \leq M\|\xi\|^{p} e^{-\lambda\left(t_{1}-t_{0}\right)}+\left(c_{3}+\psi h\right) e^{c h} \\
& \leq M\|\xi\|^{p} e^{-\lambda\left(t_{1}-t_{0}\right)}+r \\
& =\mathbb{E} V\left(x\left(t^{*}\right), t^{*}\right),
\end{aligned}
$$

which is a contradiction. Therefore, (3.4) holds and (3.3) must be true for $k=1$.

\section{Step 2:}

Now, we assume that (3.3) holds for all $k \leq m, m \in \mathbb{N}^{*}$, i.e. for $k=1,2, . ., m$

$$
\mathbb{E} V(x(t), t) \leq M\|\xi\|^{p} e^{-\lambda\left(t_{k}-t_{0}\right)}+r, t \in\left[t_{k-1}, t_{k}\right) .
$$

We will show that

$$
\mathbb{E} V(x(t), t) \leq M\|\xi\|^{p} e^{-\lambda\left(t_{m+1}-t_{0}\right)}+r, t \in\left[t_{m}, t_{m+1}\right) .
$$

Suppose (3.12) does not hold true and define $t^{*}=\inf \left\{t \in\left[t_{m}, t_{m+1}\right) ; \mathbb{E} V(x(t), t)>M\|\xi\|^{p} e^{-\lambda\left(t_{m+1}-t_{0}\right)}+\alpha\right\}$ where $\alpha=(R+\psi h) e^{c h}$ with $R=r+d$.

Thanks to conditions (ii), (iv) and inequality (3.11),

$$
\begin{aligned}
\mathbb{E} V\left(x\left(t_{m}\right), t_{m}\right) & \leq d_{m} \mathbb{E} V\left(x\left(t_{m}^{-}\right), t_{m}^{-}\right)+\rho_{m}, \\
& \leq d_{m} M\|\xi\|^{p} e^{-\lambda\left(t_{m}-t_{0}\right)}+d_{m} r+\rho_{m}, \\
& \leq M\|\xi\|^{p} e^{-\lambda\left(t_{m}-t_{0}\right)} e^{-(\lambda+c)\left(t_{m+1}-t_{m}\right)}+d_{m} r+\rho_{m}, \\
& <M\|\xi\|^{p} e^{-\lambda\left(t_{m+1}-t_{0}\right)}+d_{m} r+\rho_{m}, \\
& \leq M\|\xi\|^{p} e^{-\lambda\left(t_{m+1}-t_{0}\right)}+r+d .
\end{aligned}
$$

Thus,

$$
\mathbb{E} V\left(x\left(t_{m}\right), t_{m}\right)<M\|\xi\|^{p} e^{-\lambda\left(t_{m+1}-t_{0}\right)}+R .
$$

Now, (3.13) and the continuity of the mapping $t \in$ $\left[t_{m}, t_{m+1}\right) \mapsto \mathbb{E} V(x(t), t)$ ensure the existence of $t^{*} \in$ $\left(t_{m}, t_{m+1}\right)$ such that

$$
\begin{gathered}
\mathbb{E} V\left(x\left(t^{*}\right), t^{*}\right)=M\|\xi\|^{p} e^{-\lambda\left(t_{m+1}-t_{0}\right)}+\alpha, \\
\mathbb{E} V(x(t), t)<M\|\xi\|^{p} e^{-\lambda\left(t_{m+1}-t_{0}\right)}+\alpha, t \in\left[t_{m}, t^{*}\right) .
\end{gathered}
$$

Define

$t_{*}=\sup \left\{t \in\left[t_{0}-\tau, t^{*}\right] ; \mathbb{E} V(x(t), t) \leq d_{m} M\|\xi\|^{p} e^{-\lambda\left(t_{m}-t_{0}\right)}+R\right\}$.

Then $t_{*} \in\left[t_{m}, t^{*}\right)$ and

$$
\mathbb{E} V\left(x\left(t_{*}\right), t_{*}\right)=d_{m} M\|\xi\|^{p} e^{-\lambda\left(t_{m}-t_{0}\right)}+R
$$

$$
\mathbb{E} V(x(t), t)>d_{m} M\|\xi\|^{p} e^{-\lambda\left(t_{m}-t_{0}\right)}+R, t \in\left(t_{*}, t^{*}\right] .
$$

Fix any $t \in\left[t_{*}, t^{*}\right]$, and assume that $t+\theta \geq t_{m}$, for all $\theta \in[-\tau, 0]$, then (3.14)-(3.17) imply that

$$
\begin{aligned}
\mathbb{E} V(x(t+\theta), t+\theta) & \leq M\|\xi\|^{p} e^{-\lambda\left(t_{m+1}-t_{0}\right)}+\alpha, \\
& <M\|\xi\|^{p} e^{-\lambda\left(t+\theta-t_{0}\right)}+\alpha, \\
& \leq \gamma e^{\lambda \tau} d_{m} M\|\xi\|^{p} e^{-\lambda\left(t_{m}-t_{0}\right)}+\alpha \\
& =\gamma e^{\lambda \tau}\left(d_{m} M\|\xi\|^{p} e^{-\lambda\left(t_{m}-t_{0}\right)}+\frac{\alpha}{\gamma} e^{-\lambda \tau}\right), \\
& \leq \gamma e^{\lambda \tau} \mathbb{E} V\left(x\left(t_{*}\right), t_{*}\right), \\
& \leq q \mathbb{E} V\left(x\left(t_{*}\right), t_{*}\right) .
\end{aligned}
$$

Then,

$$
\mathbb{E} V(x(t+\theta), t+\theta)<q \mathbb{E} V(x(t), t)+\delta,
$$

for $t \in\left[t_{*}, t^{*}\right], \theta \in[-\tau, 0]$. Applying again the Itô formula on $\left[t_{*}, t^{*}\right]$ and taking into account the Razumikhintype condition (iii), we obtain

$$
\begin{aligned}
\mathbb{E} V\left(x\left(t^{*}\right), t^{*}\right)= & \mathbb{E} V\left(x\left(t_{*}\right), t_{*}\right)+\int_{t_{*}}^{t^{*}} \mathbb{E} \mathcal{L} V\left(x_{s}, s\right) d s \\
\leq & \mathbb{E} V\left(x\left(t_{*}\right), t_{*}\right)+c \int_{t_{*}}^{t^{*}} \mathbb{E} V(x(s), s) d s \\
& +\psi \Delta_{\text {sup }} .
\end{aligned}
$$

Thus,

$$
\begin{aligned}
\mathbb{E} V\left(x\left(t^{*}\right), t^{*}\right) \leq & \mathbb{E} V\left(x\left(t_{*}\right), t_{*}\right) \\
& +c \int_{t_{*}}^{t^{*}} \mathbb{E} V\left(x\left(s^{*}\right), s^{*}\right) d s+\psi h .
\end{aligned}
$$


Condition (iv), (3.14) and (3.16) and the Gronwall inequality imply

$$
\begin{aligned}
& \mathbb{E} V\left(x\left(t^{*}\right), t^{*}\right) \\
& \leq\left(\mathbb{E} V\left(x\left(t_{*}\right), t_{*}\right)+\psi h\right) e^{c\left(t^{*}-t_{*}\right)} \\
& =\left(d_{m} M\|\xi\|^{p} e^{-\lambda\left(t_{m}-t_{0}\right)}+R+\psi h\right) e^{c\left(t^{*}-t_{*}\right)}, \\
& <\left(d_{m} M\|\xi\|^{p} e^{-\lambda\left(t_{m}-t_{0}\right)}+R+\psi h\right) e^{c\left(t_{m+1}-t_{m}\right)}, \\
& \leq\left[M\|\xi\|^{p} e^{-\lambda\left(t_{m}-t_{0}\right)} e^{-(\lambda+c)\left(t_{m+1}-t_{m}\right)}+R+\psi h\right] e^{c\left(t_{m+1}-t_{m}\right)}, \\
& \leq M\|\xi\|^{p} e^{-\lambda\left(t_{m+1}-t_{0}\right)}+(R+\psi h) e^{c h} .
\end{aligned}
$$

Then,

$$
\mathbb{E} V\left(x\left(t^{*}\right), t^{*}\right)<M\|\xi\|^{p} e^{-\lambda\left(t_{m+1}-t_{0}\right)}+\alpha,
$$

which is a contradiction. Therefore (3.12) must hold true. Thus, by induction, we have obtained that (3.3) holds for all $k \in \mathbb{N}$.

\section{Step 3:}

Now, we can finish our proof. Thanks to condition $(i)$ and inequality (3.3),

$$
\begin{aligned}
\mathbb{E}|x(t)|^{p} & \leq \frac{M\|\xi\|^{p}}{c_{1}} e^{-\lambda\left(t_{k}-t_{0}\right)}+\frac{r}{c_{1}} \\
& \leq \frac{M\|\xi\|^{p}}{c_{1}} e^{-\lambda\left(t-t_{0}\right)}+\frac{r}{c_{1}}
\end{aligned}
$$

for $t \in\left[t_{k-1}, t_{k}\right), k \in \mathbb{N}$. Therefore, system (2.1) is $p$-th moment practically exponentially stable with $\eta=\frac{r}{c_{1}}$. Now, before proving our main result on almost sure practical exponential stability, we recall a result which will be crucial for our analysis and whose proof can be found in [16].

Lemma 3.2. Let $g=\left(g_{1}, \ldots, g_{m}\right) \in L^{2}\left(\mathbb{R}_{+}, \mathbb{R}^{m}\right)$, and let $T, \alpha, \beta$ be positive numbers. Then

$\mathbb{P}\left(\sup _{0 \leq t \leq T}\left[\int_{0}^{t} g(s) d W_{s}-\frac{\alpha}{2} \int_{0}^{t}|g(s)|^{2} d s\right]>\beta\right) \leq \exp (-\alpha \beta)$.

Theorem 3.3. Assume conditions of Theorem 3.1 with $p \geq 1, \Delta_{\text {sup }}=\sup _{k \in \mathbb{N}}\left\{t_{k}-t_{k-1}\right\} \leq h<\infty$ and $\Delta_{\text {inf }}=$ $\inf _{k \in \mathbb{N}}\left\{t_{k}-t_{k-1}\right\}>0$.

Assume that there exist positive constants $\beta, L$ and $\mu \geq 1$ such that, for all $(\varphi, t) \in L_{\mathcal{F}_{t}}^{p}\left([-\tau, 0], \mathbb{R}^{n}\right) \times\left[t_{0}, \infty\right)$,

$$
\mathbb{E}\left(|f(\varphi, t)|^{p}+|g(\varphi, t)|^{p}\right) \leq L \sup _{-\tau \leq \theta \leq 0} \mathbb{E}|\varphi(\theta)|^{p}+\beta .
$$

Then (3.20) implies

$$
\left|x\left(t, t_{0}, \xi\right)\right| \leq C\|\xi\|^{p} e^{-\lambda\left(t-t_{0}\right)}+\mu^{\frac{1}{p}}, \quad \forall t \geq t_{0}, \text { a.s. }
$$

In other words, the p-th moment practical exponential stability implies the almost sure practical exponential stability.
Proof. Take $0<\delta<\Delta_{\text {inf }}$ sufficiently small. For such a fixed $\delta>0$, set $k_{\delta}=\left[\frac{t_{k}-t_{k-1}}{\delta}\right] \in \mathbb{N}$. Then $k_{\delta} \leq\left[\frac{\Delta_{\text {sup }}}{\delta}\right]<$ $\infty$ and for any $t \in\left[t_{k-1}, t_{k}\right)$, there exists some $i$ with $1 \leq i \leq k_{\delta}+1$ such that $t_{k-1}+(i-1) \delta \leq t<t_{k-1}+i \delta$. Thus, for any $t \in\left[t_{k-1}, t_{k}\right), k \in \mathbb{N}$, one has

$$
\mathbb{E}\left[\sup _{t_{k-1} \leq t<t_{k}}|x(t)|^{p}\right] \leq \sum_{i=1}^{k_{\delta}+1} \mathbb{E}\left[\sup _{t_{k-1}+(i-1) \delta \leq t<t_{k-1}+i \delta}|x(t)|^{p}\right] .
$$

From [16, page 178] we have for any $a, b, c \geq 0$,

$$
(a+b+c)^{p} \leq 3^{p}\left(a^{p}+b^{p}+c^{p}\right) .
$$

For each $i$ satisfying $1 \leq i \leq k_{\delta}+1, k \in \mathbb{N}$,

$$
\begin{aligned}
\int_{t_{k-1}+(i-1) \delta}^{t} d x(s)= & \int_{t_{k-1}+(i-1) \delta}^{t} f\left(x_{s}, s\right) d s \\
& +\int_{t_{k-1}+(i-1) \delta}^{t} g\left(x_{s}, s\right) d W(s) .
\end{aligned}
$$

Thus,

$$
\begin{aligned}
x(t)= & x\left(t_{k-1}+(i-1) \delta\right)+\int_{t_{k-1}+(i-1) \delta}^{t} f\left(x_{s}, s\right) d s \\
& +\int_{t_{k-1}+(i-1) \delta}^{t} g\left(x_{s}, s\right) d W(s),
\end{aligned}
$$

and

$$
\begin{aligned}
|x(t)| \leq & \left|x\left(t_{k-1}+(i-1) \delta\right)\right|+\int_{t_{k-1}+(i-1) \delta}^{t_{k-1}+i \delta}\left|f\left(x_{s}, s\right)\right| d s \\
& +\left|\int_{t_{k-1}+(i-1) \delta}^{t} g\left(x_{s}, s\right) d W(s)\right| .
\end{aligned}
$$

Then,

$$
\begin{aligned}
|x(t)|^{p \leq} & 3^{p}\left|x\left(t_{k-1}+(i-1) \delta\right)\right|^{p} \\
& +3^{p}\left(\int_{t_{k-1}+(i-1) \delta}^{t_{k-1}+i \delta}\left|f\left(x_{s}, s\right)\right| d s\right)^{p} \\
& +3^{p}\left|\int_{t_{k-1}+(i-1) \delta}^{t} g\left(x_{s}, s\right) d W(s)\right|^{p} .
\end{aligned}
$$

Therefore

$$
\begin{aligned}
& \sup _{t_{k-1}+(i-1) \delta \leq t<t_{k-1}+i \delta}|x(t)|^{p} \leq 3^{p}\left|x\left(t_{k-1}+(i-1) \delta\right)\right|^{p} \\
& +3^{p}\left(\int_{t_{k-1}+(i-1) \delta}^{t_{k-1}+i \delta}\left|f\left(x_{s}, s\right)\right| d s\right)^{p}, \\
& +3^{p} \sup _{t_{k-1}+(i-1) \delta \leq t<t_{k-1}+i \delta}\left|\int_{t_{k-1}+(i-1) \delta}^{t} g\left(x_{s}, s\right) d W(s)\right|^{p} .
\end{aligned}
$$


Taking expectation in (3.24),

$$
\begin{aligned}
& \mathbb{E}\left[\sup _{t_{k-1}+(i-1) \delta \leq t<t_{k-1}+i \delta}|x(t)|^{p}\right] \\
& \leq 3^{p} \mathbb{E}\left(\left|x\left(t_{k-1}+(i-1) \delta\right)\right|^{p}\right) \\
& +3^{p} \mathbb{E}\left(\int_{t_{k-1}+(i-1) \delta}^{t_{k-1}+i \delta}\left|f\left(x_{s}, s\right)\right| d s\right)^{p} \\
& +3^{p} \mathbb{E}\left(\sup _{t_{k-1}+(i-1) \delta \leq t<t_{k-1}+i \delta}\left|\int_{t_{k-1}+(i-1) \delta}^{t} g\left(x_{s}, s\right) d W(s)\right|^{p}\right) .
\end{aligned}
$$

In view of (3.20), we have

$$
\mathbb{E}\left|x\left(t_{k-1}+(i-1) \delta\right)\right|^{p} \leq \frac{M\|\xi\|^{p}}{c_{1}} e^{-\lambda\left(t_{k-1}-t_{0}\right)}+\frac{r}{c_{1}} .
$$

Thanks now to (3.25), (3.26), (5.1)-(5.2), we deduce

$$
\begin{aligned}
& \mathbb{E}\left[\sup _{t_{k-1}+(i-1) \delta \leq t<t_{k-1}+i \delta}|x(t)|^{p}\right] \\
& \leq 3^{p}\left[\left(\delta^{p}+C_{p} \delta^{\frac{p}{2}}\right) L e^{\lambda \tau}+1\right] \frac{M\|\xi\|^{p}}{c_{1}} e^{-\lambda\left(t_{k-1}-t_{0}\right)}, \\
& +3^{p}\left[\frac{r}{c_{1}}+\beta \delta^{p}+C_{p} \beta \delta^{\frac{p}{2}}+\frac{L r}{c_{1}}\left(C_{p} \delta^{\frac{p}{2}}+\delta^{p}\right)\right] .
\end{aligned}
$$

Then, from (3.23) and (3.27),

$$
\begin{aligned}
\mathbb{E}\left[\sup _{t_{k-1} \leq t<t_{k}}|x(t)|^{p}\right] \\
\leq 3^{p}\left(k_{\delta}+1\right)\left[\left(\delta^{p}+C_{p} \delta^{\frac{p}{2}}\right) L e^{\lambda \tau}+1\right] \frac{M\|\xi\|^{p}}{c_{1}} e^{-\lambda\left(t_{k-1}-t_{0}\right)}, \\
\quad+3^{p}\left(k_{\delta}+1\right)\left[\frac{r}{c_{1}}+\beta \delta^{p}+C_{p} \beta \delta^{\frac{p}{2}}+\frac{L r}{c_{1}}\left(C_{p} \delta^{\frac{p}{2}}+\delta^{p}\right)\right] .
\end{aligned}
$$

Therefore,

$$
\mathbb{E}\left[\sup _{t_{k-1} \leq t<t_{k}}|x(t)|^{p}\right] \leq D^{p}\|\xi\|^{p} e^{-\lambda\left(t_{k-1}-t_{0}\right)}+\mu,
$$

where

$$
D^{p}=3^{p}\left(\frac{h}{\delta}+1\right) \frac{M}{c_{1}}\left[\left(\delta^{p}+C_{p} \delta^{\frac{p}{2}}\right) L e^{\lambda \tau}+1\right],
$$

and

$$
\mu=3^{p}\left(\frac{h}{\delta}+1\right)\left[\frac{r}{c_{1}}+\beta \delta^{p}+C_{p} \beta \delta^{\frac{p}{2}}+\frac{L r}{c_{1}}\left(C_{p} \delta^{\frac{p}{2}}+\delta^{p}\right)\right] .
$$

We will assume that $3^{p} \frac{r}{c_{1}} \geq 1$. Notice that this assumption guarantees that $\mu \geq 1$.

Let $\epsilon \in(0, \lambda)$ be arbitrary. Then Lemma 3.2 yields

$$
\mathbb{P}\left\{\sup _{t_{k-1} \leq t<t_{k}}|x(t)|^{p}>D^{p}\|\xi\|^{p} e^{-(\lambda-\epsilon)\left(t_{k-1}-t_{0}\right)}\right\} \leq e^{-\epsilon\left(t_{k-1}-t_{0}\right)} .
$$

By the Borel-Cantelli lemma we obtain

$$
\begin{aligned}
\sup _{t_{k-1} \leq t<t_{k}}|x(t)|^{p} & \leq D^{p}\|\xi\|^{p} e^{-(\lambda-\epsilon)\left(t_{k-1}-t_{0}\right)} \\
& \leq D^{p}\|\xi\|^{p} e^{-(\lambda-\epsilon)\left(t_{k-1}-t_{0}\right)}+\mu \text {, a.s., }
\end{aligned}
$$

which implies

$$
|x(t)|^{p} \leq D^{p}\|\xi\|^{p} e^{-(\lambda-\epsilon)\left(t_{k-1}-t_{0}\right)}+\mu, \quad \text { a.s },
$$

for $t \in\left[t_{k-1}, t_{k}\right), k \in \mathbb{N}$. Letting $\epsilon \rightarrow 0$ we deduce that, for any $t \in\left[t_{k-1}, t_{k}\right), k \in \mathbb{N}$,

$$
|x(t)|^{p}-\mu \leq D^{p}\|\xi\|^{p} e^{-\lambda\left(t-t_{0}\right)}, \quad \text { a.s. }
$$

We know that

$$
|x(t)|^{p}-\mu=\left(|x(t)|-\mu^{\frac{1}{p}}\right) \sum_{n=1}^{p}|x(t)|^{p-n} \mu^{\frac{n-1}{p}} .
$$

Thanks to condition $\mu \geq 1$,

$$
|x(t)|^{p}-\mu \geq\left(|x(t)|-\mu^{\frac{1}{p}}\right) .
$$

Then,

$$
|x(t)|-\mu^{\frac{1}{p}} \leq D^{p}\|\xi\|^{p} e^{-\lambda\left(t-t_{0}\right)}, \quad \forall t \geq t_{0}, \quad \text { a.s. }
$$

It thus follows

$$
|x(t)| \leq D^{p}\|\xi\|^{p} e^{-\lambda\left(t-t_{0}\right)}+\mu^{\frac{1}{p}}, \quad \forall t \geq t_{0}, \quad \text { a.s. }
$$

Therefore, system (2.1) is almost surely practically exponentially stable with $\eta=\mu^{\frac{1}{p}}$.

Remark 3.4. Observe that theorems 3.1 and 3.3 provide sufficient conditions for the practical stability of solutions both in the $p$ th-moment and pathwise. These can be interpreted as some kind of stabilization results because the problem without impulses may be unstable while the impulsive one may become practically stable. Also, when the problem is already practically stable before the impulses are taken into account, the precedent results provide sufficient conditions for the system to remain practically stable, which is a proof for the robustness of the problem.

\section{An application examples}

Example 4.1. Consider a scalar ISDDS of the form:

$\left\{\begin{aligned} d x(t) & =x(t) d t+\alpha(x(t), x(t-0.1), t) d W(t), t \neq t_{k}, t \geq 0, \\ \Delta x\left(t_{k}\right) & =-0.5 x\left(t_{k}^{-}\right), \quad k \in \mathbb{N},\end{aligned}\right.$

Then,

$$
\begin{gathered}
f\left(x_{t}, t\right)=x(t), \\
g\left(x_{t}, t\right)=\alpha\left(x_{t}(0), x_{t}(-0.1), t\right)=\alpha(x(t), x(t-0.1), t),
\end{gathered}
$$

We assume that there exists $\sigma>0$ such that $|\alpha(x, y, t)| \leq \sigma$. Then

$$
\mathbb{E}\left|f\left(x_{t}, t\right)\right|^{p}+\mathbb{E}\left|g\left(x_{t}, t\right)\right|^{p} \leq \mathbb{E}|x(t)|^{p}+\sigma^{p},
$$


Thus, condition (3.21) holds for system (4.1) with $L=1$ and $\beta=\sigma^{p}$. Let $p=2$ and $V(x, t)=x^{2}$. Therefore, the constants in Theorem 3.1 are $c_{1}=c_{2}=1, c_{3}=0.1$, $q=5, \delta=0$.

Then, we have

$$
\mathbb{E} V\left(x\left(t_{k}^{-}\right)+I_{k}\left(x\left(t_{k}^{-}\right), t_{k}\right), t_{k}\right) \leq 0.45 \mathbb{E} V\left(x\left(t_{k}^{-}\right), t_{k}\right)+0.1
$$

and

$$
\mathbb{E} \mathcal{L} V\left(x_{t}, t\right) \leq 2 \mathbb{E}|x(t)|^{2}+\sigma^{2} .
$$

Thus, $c=2, d_{k}=0.45, d=0.1=\rho_{k}$ and $\psi=\sigma^{2}$. We choose $t_{k}-t_{k-1}=h=0.2, \lambda=0.01$ and $\gamma=2.5$. Then,

$$
c_{3} \gamma e^{\lambda \tau}=0.25
$$

and we choose $\psi=\sigma^{2}=0.3$,

$$
\left(\psi h+c_{3}\right) e^{c h}=0.238 .
$$

Thus, we can pick $r=0.24$.

Then, all assumptions in the previous two theorems are fulfilled. Therefore, system (4.1) is mean square (i.e. second moment) practically exponentially stable with $\eta=\frac{r}{c_{1}}=0.24$.

Now, we exhibit the following numerical example

Example 4.2. Consider the linear impulsive stochastic delay system

$$
\left\{\begin{aligned}
d x(t)= & (A x(t)+B x(t-1)) d t+(C x(t) \\
& +D x(t-1)) d W(t), t \neq t_{k}, t \geq 0 \\
\Delta x\left(t_{k}\right)= & -0.4 x\left(t_{k}^{-}\right), \quad k \in \mathbb{N}^{*}
\end{aligned}\right.
$$

where $A=\left(\begin{array}{cc}-0.4 & 0 \\ 0 & -0.4\end{array}\right), B=\left(\begin{array}{ll}1 & 0 \\ 0 & 1\end{array}\right), C=$ $\left(\begin{array}{cc}0.9 & 0 \\ 0 & 0.9\end{array}\right), D=\left(\begin{array}{ll}1 & 0 \\ 0 & 1\end{array}\right), x(t)=\left(x_{1}(t), x_{2}(t)\right)^{T}$. Then, $f(x(t), x(t-1), t)=(A x(t)+B x(t-1)), g(x(t), x(t-$ $1), t)=(C x(t)+D x(t-1))$ and $I_{k}\left(x\left(t_{k}^{-}\right), t_{k}\right)=-0.4 x\left(t_{k}^{-}\right)$. We assume that $\|A\|=\sqrt{\lambda_{\text {max }}\left(A^{T} A\right)}$ for all $A \in \mathcal{M}_{2}(\mathbb{R})$. Let $p=2$ and $V(x, t)=|x|^{2}$. Then,

$$
\begin{aligned}
& \mathbb{E}|f(x(t), x(t-1), t)|^{2}+\mathbb{E}|g(x(t), x(t-1), t)|^{2} \\
& \leq 2\left(\|A\|^{2}+\|C\|^{2}\right) \mathbb{E}|x(t)|^{2}+2\left(\|B\|^{2}+\|D\|^{2}\right) \mathbb{E}|x(t-1)|^{2}, \\
& \leq 1.7 \mathbb{E}|x(t)|^{2}+4 \mathbb{E}|x(t-1)|^{2}+0.4, \\
& \leq 4\left(\mathbb{E}|x(t)|^{2}+\mathbb{E}|x(t-1)|^{2}\right)+0.4 .
\end{aligned}
$$

Therefore, condition (3.21) holds for system (4.1) with $L=4$ and $\beta=0.4$. Let $p=2$ and $V(x, t)=|x|^{2}$. Then, the constants in Theorem 3.1 are $c_{1}=c_{2}=1, c_{3}=0.3$, $q=6.12, \delta=0$.

Consequently,

$$
\mathbb{E} V\left(x\left(t_{k}^{-}\right)+I_{k}\left(x\left(t_{k}^{-}\right), t_{k}\right), t_{k}\right) \leq 0.6 \mathbb{E} V\left(x\left(t_{k}^{-}\right), t_{k}\right)+0.3
$$

and

$$
\begin{aligned}
& \mathbb{E} \mathcal{L} V\left(x_{t}, t\right) \\
& \leq 2 \mathbb{E}\left(x(t)^{T} f(x(t), x(t-1), t)\right)+\mathbb{E}|g(x(t), x(t-1), t)|^{2}, \\
& \leq 2 \mathbb{E}\left(x(t)^{T} A x(t)\right)+2 \mathbb{E}\left(x(t)^{T} B x(t-1)\right)+\mathbb{E}|g(x(t), x(t-1), t)|^{2}, \\
& \leq 2 \mathbb{E}|x|^{2}\left(\|A\|^{2}+\|B\|^{2}\right)+4 \mathbb{E}|x|^{2}, \\
& \leq 6.08 \mathbb{E}(V(x, t))+0.5 .
\end{aligned}
$$

Thus, $c=6.08, d_{k}=0.6, d=0.5=\rho_{k}$ and $\psi=0.5$. We choose $t_{k}-t_{k-1}=h=0.2, \lambda=0.02$ and $\gamma=6$. Then,

$$
c_{3} \gamma e^{\lambda \tau}=1.83
$$

and

$$
\left(\psi h+c_{3}\right) e^{c h}=1.348 .
$$

Thus, we can choose $r=1.35$.

Then, all the assumptions of the previous two theorems are fulfilled. Therefore, system (4.2) is mean square practically exponentially stable with $\eta=\frac{r}{c_{1}}=1.35$.

\section{Appendix}

Lemma 5.1. Under conditions in Theorem 3.1 and Theorem 3.3 , we define

$$
\begin{gathered}
J_{1}=\mathbb{E}\left(\int_{t_{k-1}+(i-1) \delta}^{t_{k-1}+i \delta}\left|f\left(x_{s}, s\right)\right| d s\right)^{p}, \\
J_{2}=\mathbb{E}\left(\sup _{t_{k-1}+(i-1) \delta \leq t<t_{k-1}+i \delta}\left|\int_{t_{k-1}+(i-1) \delta}^{t} g\left(x_{s}, s\right) d W(s)\right|^{p}\right) .
\end{gathered}
$$

Then

$$
J_{1} \leq L \delta^{p} e^{\lambda \tau} \frac{M\|\xi\|^{p}}{c_{1}} e^{-\lambda\left(t_{k-1}-t_{0}\right)}+\delta^{p}\left(\beta+\frac{L r}{c_{1}}\right)
$$

and

$$
J_{2} \leq L C_{p} \delta^{\frac{p}{2}} \frac{M\|\xi\|^{p}}{c_{1}} e^{\lambda \tau} e^{-\lambda\left(t_{k-1}-t_{0}\right)}+C_{p} \beta \delta^{\frac{p}{2}}+\frac{L r}{c_{1}} C_{p} \delta^{\frac{p}{2}} .
$$


Proof. By (3.20), (3.21) and the Hölder inequality,

$$
\begin{aligned}
J_{1} & \leq \mathbb{E}\left[\left(\int_{t_{k-1}+(i-1) \delta}^{t_{k-1}+i \delta} d s\right)^{p-1}\left(\int_{t_{k-1}+(i-1) \delta}^{t_{k-1}+i \delta}\left|f\left(x_{s}, s\right)\right|^{p} d s\right)\right], \\
& \leq \delta^{p-1} \mathbb{E}\left(\int_{t_{k-1}+(i-1) \delta}^{t_{k-1}+i \delta}\left|f\left(x_{s}, s\right)\right|^{p} d s\right), \\
& \leq \delta^{p-1} \int_{t_{k-1}+(i-1) \delta}^{t_{k-1}+i \delta}\left(L \sup _{-\tau \leq \theta \leq 0} \mathbb{E}|x(s+\theta)|^{p}+\beta\right) d s, \\
& \leq L \delta^{p-1} \int_{t_{k-1}+(i-1) \delta-\tau \leq \theta \leq 0}^{t_{k-1}+i \delta} \sup _{-} \mathbb{E}|x(s+\theta)|^{p} d s+\beta \delta^{p}, \\
& \leq L \delta^{p-1} \frac{M\|\xi\|^{p}}{c_{1}} \int_{t_{k-1}+(i-1) \delta}^{t_{k-1}+i \delta} e^{-\lambda\left(s-\tau-t_{0}\right)} d s+\beta \delta^{p}+\frac{L r}{c_{1}} \delta^{p}, \\
& \leq L \delta^{p} \frac{M\|\xi\|^{p}}{c_{1}} e^{-\lambda\left(t_{k-1}+(i-1) \delta-\tau-t_{0}\right)}+\delta^{p}\left(\beta+\frac{L r}{c_{1}}\right) .
\end{aligned}
$$

Then,

$$
J_{1} \leq L \delta^{p} e^{\lambda \tau} \frac{M\|\xi\|^{p}}{c_{1}} e^{-\lambda\left(t_{k-1}-t_{0}\right)}+\delta^{p}\left(\beta+\frac{L r}{c_{1}}\right) .
$$

On the other hand, by the Burkholder-Davis-Gundy inequality and Hölder inequality, we have

$$
\begin{aligned}
J_{2} & \leq C_{p} \mathbb{E}\left(\int_{t_{k-1}+(i-1) \delta}^{t_{k-1}+i \delta}\left|g\left(x_{s}, s\right)\right|^{2} d s\right)^{\frac{p}{2}} \\
& \leq C_{p} \delta^{\frac{p}{2}-1} \mathbb{E}\left(\int_{t_{k-1}+(i-1) \delta}^{t_{k-1}+i \delta}\left|g\left(x_{s}, s\right)\right|^{p} d s\right),
\end{aligned}
$$

where $C_{p}>0$ is a constant which only depends on $p$. From (3.21), for all $(\varphi, t) \in L_{\mathcal{F}_{t}}^{p}\left([-\tau, 0], \mathbb{R}^{n}\right) \times\left[t_{0}, \infty\right)$, we have

$$
\mathbb{E}|g(\varphi, t)|^{p} \leq L \sup _{-\tau \leq \theta \leq 0} \mathbb{E}|\varphi(\theta)|^{p}+\beta .
$$

By inequality (5.4),

$$
\begin{aligned}
J_{2} & \leq C_{p} \delta^{\frac{p}{2}-1} \int_{t_{k-1}+(i-1) \delta}^{t_{k-1}+i \delta}\left(L \sup _{-\tau \leq \theta \leq 0} \mathbb{E}|x(s+\theta)|^{p}+\beta\right) d s, \\
& \leq L C_{p} \delta^{\frac{p}{2}-1} \int_{t_{k-1}+(i-1) \delta}^{t_{k-1}+i \delta} \sup _{-\tau \leq \theta \leq 0} \mathbb{E}|x(s+\theta)|^{p} d s+C_{p} \beta \delta^{\frac{p}{2}} .
\end{aligned}
$$

Thus,

$$
J_{2} \leq L C_{p} \delta^{\frac{p}{2}} \frac{M\|\xi\|^{p}}{c_{1}} e^{\lambda \tau} e^{-\lambda\left(t_{k-1}-t_{0}\right)}+C_{p} \beta \delta^{\frac{p}{2}}+\frac{L r}{c_{1}} C_{p} \delta^{\frac{p}{2}}
$$

Acknowledgements. Partially supported by FEDER and Ministerio de Economía y Competitividad (Spain) grant MTM2015-63723-P, and by Proyecto de Excelencia P12-FQM-1492 (Junta de Andalucía).

We would like to thank the referees and the associate editor for the interesting and helpful suggestions which allowed us to improve the presentation of this paper.

\section{References}

[1] T. Caraballo, M. A. Hammami, L. Mchiri, Practical asymptotic stability of nonlinear stochastic evolution equations, Stochastic Analysis and Applications, 32 (2014), 77-87.

[2] T. Caraballo, M. A. Hammami, L. Mchiri, Practical exponential stability in mean square of stochastic partial differential equations, Collectanea mathematica, doi: 10.1007/s13348-0140124-9.

[3] T. Caraballo, M. A. Hammami, L. Mchiri, On the practical global uniform asymptotic stability of stochastic differential equations, Stochastics, 88 (2016), No. 1, 45-56

[4] T. Caraballo, K. Liu, A. Truman, Stochastic functional partial differential equations: existence, uniqueness and asymptotic decay property, Proc. R. Soc. London A 456 (2000), 1775-1802.

[5] P. Cheng, F. Deng, Global exponential stability of impulsive stochastic functional differential systems. Statistics and Probability Letters 80 (2010), 1854-1862.

[6] M. Dlala, M. A. Hammami, Uniform exponential practical stability of impulsive perturbed systems Journal of Dynamical and Control Systems 13 (2007), No. 3, 373-386.

[7] H. Bao, J. Cao, Exponential stability for stochastic BAM networks with discrete and distributed delays. Applied Mathematics and Computation 218 (2012), 61886199.

[8] H. Bao, J. Cao, Existence and uniqueness of solutions to neutral stochastic functional differential equations with infinite delay Applied Mathematics and Computation 215 (2009), 17321743.

[9] H. Bao, J. Cao, Stochastic global exponential stability for neutral-type impulsive neural networks with mixed time-delays and Markovian jumping parameters Commun Nonlinear Sci Numer Simulat 16 (2011), 37863791.

[10] J. Ding, J. Cao, G. Feng, J. Zhou, A. Alsaedi, A. Al-Barakati, H. M. Fardoun, Exponential synchronization for a class of impulsive networks with time-delays based on single controller $\mathrm{Neu}$ rocomputing 218 (2016), 113119.

[11] V. Lakshmikantham, D. Baïnov, P. Simeonov, Theory of impulsive differential equations. World Scientific Publishing, 1989.

[12] J. Liu, X. Liu, W-C. Xie, Impulsive stabilization of stochastic functional differential equations. Applied Mathematics Letters, 24, 264-269, (2011).

[13] J. Liu, X. Liu, W.-C. Xie, Existence and uniqueness results for impulsive hybrid stochastic delay systems, Communications on Applied Nonlinear Analysis 17 (2010), 37-54.

[14] X. Mao, Razumikhin-type theorems on exponential stability of stochastic functional differential equations. Stoch. Process. Appl. 65 (1996), 233-250.

[15] X. Mao, Exponential Stability of Stochastic Differential Equations, Marcel Dekker, Inc.: New York, 1994.

[16] X. Mao, Stochastic Differential Equations and Applications, Ellis Horwood, Chichester, U.K, 1997.

[17] S. E. A. Mohamed, Stochastic functional differential equations, Longman Scientific and technical, New York, 1986.

[18] S. Peng, Y. Zhang, Razumikhin-Type Theorems on th Moment Exponential Stability of Impulsive Stochastic Delay Differential Equations. IEEE Transactions on automatic control, Vol. 55, No. 8, August 2010, 1917-1922.

[19] S. Yi, L. Xiaoxin, Razumikhin-type theorems on exponential stability of neutral stochastic functional differential equations. Chinese Science Bulletin Vol .44 No. 24 December 1999, 22252228. 